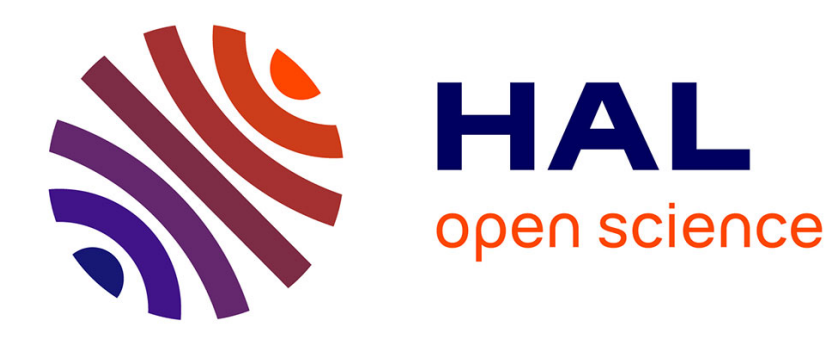

\title{
Un système d'arrosage multivoie quantitatif
}

Alain Gerbaud, Alain Daguenet

\section{To cite this version:}

Alain Gerbaud, Alain Daguenet. Un système d'arrosage multivoie quantitatif. Agronomie, 1984, 4 (10), pp.1009-1012. hal-00884605

\section{HAL Id: hal-00884605 https://hal.science/hal-00884605}

Submitted on 1 Jan 1984

HAL is a multi-disciplinary open access archive for the deposit and dissemination of scientific research documents, whether they are published or not. The documents may come from teaching and research institutions in France or abroad, or from public or private research centers.
L'archive ouverte pluridisciplinaire HAL, est destinée au dépôt et à la diffusion de documents scientifiques de niveau recherche, publiés ou non, émanant des établissements d'enseignement et de recherche français ou étrangers, des laboratoires publics ou privés. 


\title{
Un système d'arrosage multivoie quantitatif
}

\author{
Alain GERBAUD \& Alain DAGUENET $(*)$ \\ I.N.R.A., Laboratoire de Chimie Biologique et de Photophysiologie, I.N.A.P.G. (centre de Grignon), \\ F 78850 Thiverval-Grignon \\ (*) Service de Radioagronomie, Département de Biologie, C.E.N. de Cadarache, B.P. I, \\ F 13115 Saint-Paul-Lez-Durance
}

RESUMÉ

On a réalisé un système d'arrosage multivoie réalisable économiquement en laboratoire et dont la précision est nettement supérieure à celle des systèmes traditionnels. Des réservoirs correspondants à chaque voie sont remplis, le niveau est ajusté par un trop-plein et le vidage, par un siphon, est déclenché par l'envoi d'air comprimé.

Mots clés additionnels : Arrosage automatique, stress hydrique.

A quantitative multichannel watering system.

A system for multichannel watering of plants has been developed. It was inexpensive to construct in the laboratory and was more accurate and reliable than usual systems. First water reservoirs corresponding to each channel are filled, then levels are set by overflow-pipes, and finally the reservoirs are emptied through siphons into the plant pots by means of compressed air.

Additional key words : Water limitation, water stress.

\section{INTRODUCTION}

Les conditions de culture des plantes étudiées au laboratoire, avant et pendant les expériences, influencent notablement les résultats obtenus, et il est souhaitable de disposer de lots aussi homogènes que possible ainsi que de pouvoir fixer les conditions climatiques dans un large domaine et de façon reproductible.

Les paramètres physiques les plus importants - lumière, température, concentration du $\mathrm{CO}_{2}-$ sont assez bien maîtrisés, mais l'arrosage reste fréquemment un point faible, car il est difficile de le doser avec précision pour un grand nombre de plantes simultanément.

Le manque d'un bon système d'arrosage conduit souvent les expérimentateurs à n'utiliser que des plantes arrosées à saturation ou pas du tout, ou bien les situations d'arrosage limitant, nécessaires dans l'étude des stress hydriques, sont réalisées à la main.

Il existe pourtant actuellement, d'une part, des systèmes précis (pompes doseuses, régulateurs de débit, seringues automatiques) mais qui ne sont pas adaptés à l'arrosage de lots de plantes, d'autre part, des systèmes de répartition des débits (branchements et goutteurs), souvent utilisés parce qu'ils sont économiques mais qui sont peu précis et peu fiables parce que les débits sont très sensibles aux perturbations (pression, température, poussière, entartrage).

Le montage que nous présentons ici cherche à concilier l'économie et la précision. Il est réalisable avec les moyens de chaque laboratoire.

\section{PRINCIPE DE FONCTIONNEMENT}

Le système (fig. 1) comprend autant de réservoirs qu'il y a de pots à arroser. Les réservoirs sont remplis par l'eau montant d'un bidon mis sous pression à l'air comprimé.

Le volume d'eau admis dans chaque réservoir n'est pas déterminé avec précision à cette phase ; l'ajustage du volume se fait par écoulement de l'excédent par un trop-plein. Le trop-plein est réglable en hauteur, ce qui permet un réglage individualisé du volume d'arrosage.

Quand les excédents se sont écoulés, l'arrosage proprement dit est déclenché par l'envoi d'une légère surpression d'air comprimé dans le réservoir, ce qui provoque l'amorçage d'un siphon qui vide ensuite la totalité du réservoir dans le pot correspondant.

Ces différentes opérations peuvent être commandées par un ordinateur ou un programmateur de 


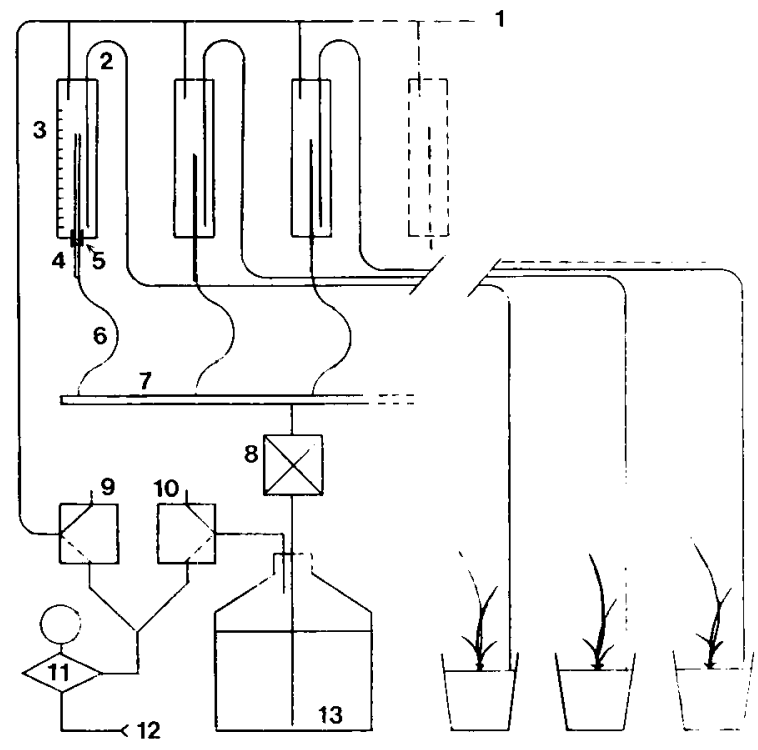

Figure 1

Schéma de l'ensemble du système d'arrosage.

I) Rampe de distribution d'air comprimé pour l'amorçage des siphons.

2) Siphon de vidange des réservoirs.

3) Réservoir gradué.

4) Tube de remplissage servant aussi de trop-plein.

5) Joint coulissant.

6) Tuyau de jonction souple.

7) Rampe de distribution d'eau.

8) Electrovanne 2 voies pour le passage de l'eau.

9) Electrovanne 3 voies mettant les réservoirs en communication soit avec l'air libre $(R)$ soit avec l'air comprimé.

10) Electrovanne 3 voies mettant la réserve d'eau en communication soit avec l'air libre (repos) soit avec l'air comprimé.

II) Détendeur réglé à $200 \mathrm{mb}$.

12) Source d'air comprimé

13) Bidon de réserve d'eau (ou de solution nutritive).

Diagram of the watering system.

1) Compressed air ramp for the priming of the siphons.

2) Siphon for the emptying of the reservoirs.

3) Graduated reservoirs.

4) Filling and overflow pipe.

5) Sliding joint.

6) Flexible tubing

7) Water distribution ramp.

8) Electric two-way valve for water.

9) Electric three-way valve connecting the reservoirs either to the compressed air or to free air.

10) Electric three-way valve connecting the water reserve either to the compressed air or to free air

11) Pressure reducer set to 200 mbar.

12) Compressed air source.

13) Water (or nutrient solution) reserve tank.

séquences, qui envoie la tension successivement, pendant des durées déterminées, aux différentes vannes du système. Le schéma d'un programmateur simple à relais est donné en annexe. En pratique l'arrosage comprend 4 phases :

\section{Phase 1:}

L'admission de l'eau dans les réservoirs se fait par la commutation de la vanne (fig. $1 \mathrm{n}^{\circ} 10$ ), qui envoie l'air comprimé dans le bidon de réserve, et de la vanne (8) qui relie le bidon aux réservoirs.

\section{Phase 2 :}

On referme les vannes. Les niveaux s'égalisent dans les différents réservoirs par le principe des vases communicants.

\section{Phase 3 :}

En laissant la vanne 10 au repos - le bidon d'eau est relié à l'air libre - on ouvre la vanne 8 , ce qui permet à l'excédent d'eau des réservoirs de s'écouler vers les bidons, jusqu'à atteindre le niveau des troppleins.

\section{Phase 4 :}

On referme la vanne d'eau (8) et on ouvre la vanne de « chasse » (9), qui précédemment mettait le haut des réservoirs en communication avec l'air libre et qui va maintenant admettre l'air comprimé. La pression fait monter l'eau dans les siphons qui s'amorcent et vident les réservoirs jusqu'au fond.

\section{AVANTAGES}

Par son principe, ce système permet d'obtenir une bonne reproductibilité et un réglage facile de la quantité d'eau versée, puisque celle-ci ne dépend ni d'une durée, ni d'un débit, ni d'une pression, paramètres dont le réglage est difficilement fiable. Le volume versé est la différence entre le volume restant après écoulement du trop-plein et le volume restant au moment où le siphon se désamorce.

Cette différence dépend de la viscosité et de la tension superficielle du liquide et des caractéristiques géométriques du réservoir qui ne varient pas puisqu'aucune pièce n'est en mouvement.

Les siphons ont un double usage : d'une part, ils permettent de remplir les réservoirs sans déclencher l'arrosage, ce qui évite de devoir poser une vanne sur chaque canal d'arrosage. D'autre part, l'arrosage peut être déclenché par une arrivée d'air comprimé commune à tous les réservoirs. Une vanne 2 voies pour l'eau et 2 vannes 3 voies pour l'air suffisent, quel que soit le nombre de voies d'arrosages du système.

Le réglage du volume d'arrosage se fait très simplement en amenant le tube du trop-plein au niveau de la graduation correspondante du réservoir. Le zéro peut être ajusté en faisant coulisser le tube du siphon.

Si on utilise un détecteur de niveau pour arrêter la montée de l'eau ( $1^{\text {re }}$ phase), la précision de la durée des phases n'a aucune importance, pourvu qu'elles soient assez longues, de l'ordre de $2 \mathrm{mn}$ par phase dans le cas de notre montage. La longueur des tuyaux reliant les réservoirs aux pots à arroser peut être quelconque. Dans une large mesure leur résistance n'influe pas sur l'arrosage.

\section{RÉALISATION PRATIQUE}

Les réservoirs (fig. 2) sont des tubes de verre sciés à la longueur en fonction du volume d'arrosage désiré. Ils sont bouchés en haut par des bouchons prépercés en caoutchouc. Comme le perçage de ces bouchons fait une surface grossière et déchire souvent le caoutchouc, l'étanchéité du passage du siphon et de l'arrivée d'air comprimé est renforcée en enfilant le tube métallique dans une gaine en tuyau silicone faisant joint. 
Le passage coulissant du trop-plein, en bas du réservoir, est réalisé à l'aide de tube à vide et d'un joint torique maintenu par 2 bouts de tube plastique rigide (fig. 2). Les trop-pleins, les siphons et les arrivées d'air sont en acier inox au départ des réservoirs, les longueurs de tuyaux sont en rilsan, en nylon ou en caoutchouc silicone.

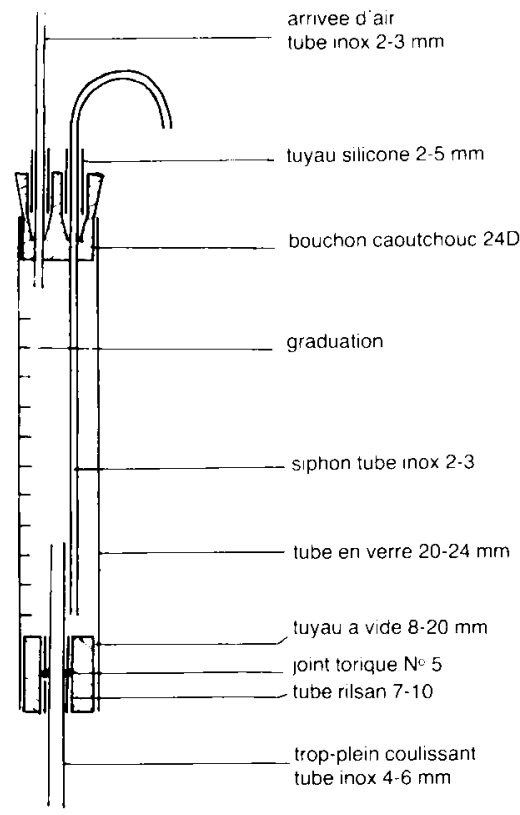

Figure 2

Construction d'un réservoir.

Diagram of a reservoir.

Toutes les connexions entre tuyaux et avec les rampes de distribution sont réalisées à l'aide de segments de tuyau en caoutchouc silicone (fig. 3). Ce mode de jonction est plus rapide, plus facile et plus solide que le collage.

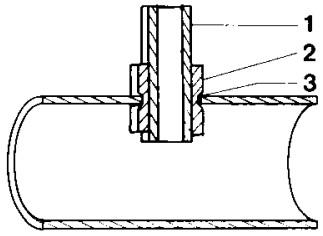

Figure 3

Détail de la jonction entre un tube et la rampe de distribution.

1) Tuyau rilsan $4 \times 6$

2) Tuyau silicone $5 \times 8$

3) Percement $\varnothing 8 \mathrm{~mm}$.

Detail of the junction between a tube and the distribution ramp.

1) Plastic tubing $4 \times 6 \mathrm{~mm}$

2) Silicone rubber pipe $5 \times 8 \mathrm{~mm}$.

3) Hole $\varnothing 8 \mathrm{~mm}$.

La pression d'air nécessaire à la montée de l'eau et à l'amorçage des siphons est voisine de $200 \mathrm{mb}$. Un seul détendeur suffit pour les 2 fonctions.

La vitesse des opérations est limitée par la lenteur des débits d'eau à la montée et à l'écoulement des excédents. Il est souhaitable que ce soit le diamètre de la vanne d'eau qui soit limitant, pour que la montée ou la descente de l'eau se répartisse également entre tous les réservoirs. Les réservoirs sont alignés horizontalement sur un panneau. Tout défaut de fonctionnement du système, défaut de remplissage ou de vidange des réservoirs, etc..., est visible immédiatement. Le détendeur et les vannes sont groupés sur un panneau indépendant.

\section{APPLICATION. PRÉCISION OBTENUE}

Nous avons testé notre prototype avec 40 réservoirs répartis sur 2 panneaux d'arrosage, correspondant chacun à une chambre de culture.

Les quantités d'eau délivrées étaient mesurées par pesée. Le volume maximum des réservoirs étant $50 \mathrm{ml}$, nous avons fait des essais en réglant le volume d'arrosage soit à 25 , soit à $35 \mathrm{ml}$.

Dans les 2 cas la reproductibilité de l'arrosage était voisine de $0,3 \mathrm{ml}$ (écart-type). La dispersion entre les voies d'arrosage était de $0,5 \mathrm{ml}$ (écart-type) au $1^{\text {er }}$ essai et $0,2 \mathrm{ml}$ après l'ajustage du niveau des siphons dans chaque réservoir.

Reçu le 20 avril 1984. Accepté le 27 juin 1984.

ANNEXE

SCHÉMA DU PROGRAMMATEUR DE SÉQUENCES

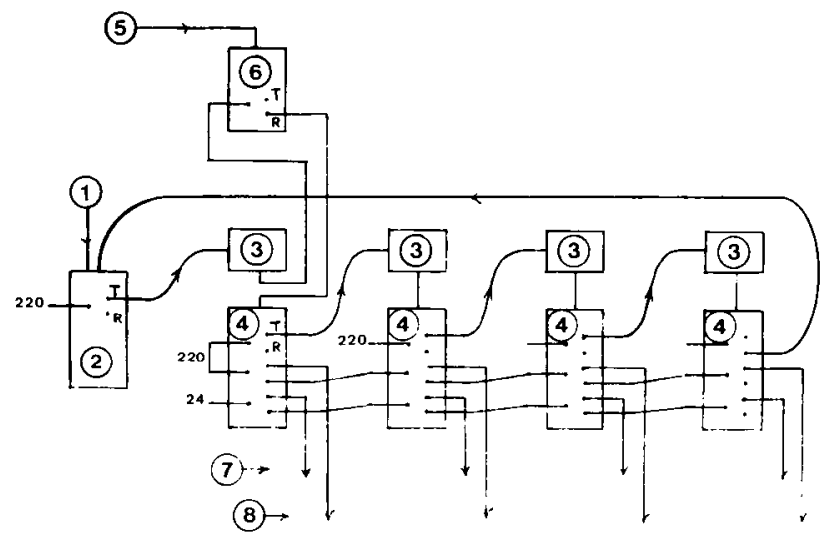

1 - Entrée du signal d'initialisation de l'arrosage $(220 \mathrm{~V})$.

2 - Relais d'initialisation (TEC $1301220 \mathrm{VAC}$ )

3 - Bloc temporisé (TEC 1832 « $\mathrm{O}$ » $220 \mathrm{VAC}, 60$ à $500 \mathrm{~s}$ ).

4 - Relais 4RT 10 A (TEC $1753220 \mathrm{VAC}$ ).

5 - Détecteur de niveau.

6 - Relais du détecteur de niveau.

7 - Sorties $24 \mathrm{~V}$.

8 - Sorties $220 \mathrm{~V}$.

Les 4 couples de sorties correspondent aux 4 phases de l'arrosage Les électrovannes 8 et 10 sont toutes les 2 actionnées pendant la phase 1. Elles doivent être branchées sur 2 sorties indépendantes du $1^{\text {er }}$ relais (le schéma ne représente qu'une seule sortie de chaque type $24 \mathrm{~V}$ ou $220 \mathrm{~V}$ ). 
REMERCIEMENTS

La rédaction et les auteurs des articles parus dans le volume 4 d'«Agronomie » qui s'achève avec le présent fascicule remercient très vivement les personnalités scientifiques qui ont accepté d'être lecteurs :

S. Adamovicz (Antibes), A. Agot (Grignon), C. Alabouvette (Dijon), J. Alboly (Versailles), J. Amoros (Rennes), H. ARN (Wädenswil), P. Atger (Versailles), H. Audemard (Avignon), P. Auriau (Versailles), J. M. Azaïs (Versailles), H. BANnerot (Versailles), P. Barnols (Clermont-Ferrand), G. Barralis (Dijon), Y. Barrière (Lusignan), G. Benz (Zürich), R. Bernhard (Bordeaux), E. Berninger (Fréjus), C. Bigot (Versailles), Y. Birot (Orléans), D. Blanc (Antibes), R. Blanchet (Toulouse), G. BoCQuier (Paris), C. Bodet (Clermont-Ferrand), P. Boissard (Versailles), P. Boistard (Toulouse), P. Bonnet (Antibes), M. Bournerias (Paris), A. Boyat (Montpellier), L. Brader (La Haye), G. BranLARD (Clermont-Ferrand), J. BRENIÈRE (Montpellier), C. BRIAN (Bordeaux), H. BRISSE (Strasbourg), A. BRÖNNIMANN (Zürich), R. Calvet (Grignon), P. Cambier (Versailles), J. M. Cases (Nancy), G. CatrouX (Dijon), A. Cauderon (Paris), Y. Cauderon (Versailles), J. C. CAyrol (Antibes), P. Champagnat (ClermontFerrand), M. Chartier (Versailles), P. Chassin (Versailles), R. Chaussod (Dijon), J. Chery (Montpellier), J. Chevaugeon (Orsay), E. Choppin de Janvry (Paris), J. Cohat (Landerneau), R. J. Cook (Pulman), J. M. Cornuet (Avignon), P. Cornuet (Versailles), C. Costes (Grignon), R. Coutin (Versailles), J. DF Crécy (Dijon), P. Cruiziat (Clermont-Ferrand), A. Dalmasso (Antibes), J. Damagnez, F. A. Daudet (Clermont-Ferrand), C. A. Dedryver (Rennes), J. Delas (Bordeaux), Y. Demari.y (Orsay), J. B. Denis (Versailles), M. Derieux (Péronne), C. Des. CoIns (Saint-Rémy-les-Chevreuse), J. C. DEvergne (Antibes), A. Dewar (Rothamsted), C. DorÉ (Versailles), R. DOuCÉ, R. Dumas de Vaulx (Avignon), P. Du Merle (Avignon), J. Dunez (Bordeaux), M. Duron (Angers), R. ECOCHARD (Toulouse), S. Essad (Versailles), J. R. Esteban-Duran (Madrid), J. C. Fardeau (Cadarache), J. Fargues (La Minière), G. Fauvel (Montpellier), A. Fleury (Grignon), G. FouillouX (Versailles), J. P. FrÉTEAUd (Montpellier), L. GACHON (Clermont-Ferrand), J. GaudillèrF (Grignon), G. Genier (Lusignan), R. E. Geraert (Gand), C. GÉrI (Orléans), E. Germain (Bordeaux), S. Gianinazzi (Dijon), D. GiN DRAT (Changins-Nyon), M. C. GIRARD (Grignon), G. Gosse (Ver- sailles), R. Goujet (La Minière), P. Grison (La Minière), D. Grouet (Versailles), P. GuY (Lusignan), A. Herbillon (Louvain), Y. Hervé (Rennes), J. C. Huguet (Avignon), B. Itier (Versailles), P. JACQUARD (Montpellier), J. JAHIER (Rennes), P. JAUZEIN (Versailles), L. Jestin (Clermont-Ferrand), P. Jourdheull (Antibes), F. Jull. IAN (Avignon), C. JUSTE (Bordeaux), F. KAAN (Montpellier), N. Kateru (Versailles), G. Labonne (Antilles), C. LamarQue (Versailles), G. LATteur (Gembloux), P. Laviolette (Villeurbanne), F. LeCLant (Montpellier), Y. LE DeunfF (Paris), J. Le Guen (Rennes), J. M. Lemaire (Avignon), M. Lemattre (Versailles), C. LESAINT (Versailles), P. LoISEAU (Clermont-Ferrand), P. Louguet (Paris VI), J. Louveau (Bures), J. M. Machet (Laon), C. Maertens (Toulouse), G. Manachere (Lyon), H. Manichon (Paris-Grignon), P. Mansat (Lusignan), J. Margara (Versailles), Y. MaUry (Versailles), A. MERrien (Toulouse), C. M. Messiaen (Antilles), J. Meynet (Fréjus), C. Montant, F. DE MONTARD (Clermont-Ferrand), J. Morizet (ClermontFerrand), G. Morvan (Avignon), M. Niqueux (Clermont-Ferrand), J. C. Onillon (Versailles), P. Pauvert (Versailles), P. Pécaut (Avignon), G. Pedro (Versailles), G. Pelletier (Versailles), H. PÉzerat (Paris), J. PICARD (Dijon), E. Pochard (Avignon), M. Pollaczek (Clermont-Ferrand), R. Pouget (Bordeaux), J. L. Puech (Toulouse), B. Rat (Angers), A. Ravisé (Bondy), G. RaYNAL (Grignon), J. C. RÉMY (Laon), M. RENARD (Rennes), G. RiBA (Guyancourt), R. RIEUX (Avignon), C. RIOU (Versailles), M. Ritier (Antibes), M. Rives (Paris), M. Robelin (Clermont-Ferrand), M. Rougrer (Versailles), P. Rousselle (Rennes), F. RouXEL (Rennes), P. Rouze (Versailles), M. Russo (Bari), W. E. SACKSTON (Québec), P. Saglio (Bordeaux), G. Salesses (Bordeaux), L. SaL ZAC (Montpellier), R. SAMSON (Angers), B. SAugIer (Orsay), J. Schmit (Versailles), P. G. Schoch (Avignon), B. SEckser (Bâle), C. Scotto, La Massese (Antibes), M. SEbillotte (Paris-Grignon), P. Signoret (Montpellier), S. Siljak-Yacovlev (orsay), G. Soulas (Dijon), G. Sposito (Riverside), H. Steiner (Stuttgart), A. TABBAGH (Pouilly s/Loire), D. TCHOUbar (Orléans), C. TENDILle (Versailles), H. Thiellement (Gif), R. Tramier (Antibes), M. Trottet (Rennes), M. Van Grunderbeck (Bordeaux-Mérignac), M. H. VAN REGENMORTEL (Strasbourg), N. VARTANian (Orsay), M. Vauclin (Grenoble), F. VeAR (Clermont-Ferrand), J. Verdier (Toulouse), A. VeY (Saint-Christol-les-Alès), O. DE Villele (Avignon), A. VinCENT (Dijon), P. VinColRT (Lusignan), A. VuITtenez (Colmar), R. WAGNER (Montpellier).

\section{Informations}

Ph. Duchaufrour. - Pédologie. 1. Pédogenèse et Classification. $2^{\mathrm{e}}$ édition revue et augmentée, Paris, Masson Ed., 1983, 491 p.

Rappelons qu'au Précis de Pédologie de $\mathrm{Ph}$. DUCHAUFFOUR, désormais épuisé, a succédé, à partir de 1977, un ensemble de deux volumes, publiés sous la direction de Ph. Duchauffour et B. SOUCHIER, sous le titre général de «Pédologie »:

Le premier (1977), sous titré « Pédogenèse et Classification ", dû à Ph. Duchauffour lui-même; le second, rédigé par de nombreux auteurs coordonnés par M. BONNE.AU et B. SOUCHIER (1979), consacré aux « Constituants et propriétés du sol ».

Il s'agit ici d'une nouvelle édition du tome 1 . Comme la précédente, elle comporte 2 parties :

La première consacrée aux processus physicochimiques de la pédogenèse traite successivement de l'évolution de la fraction minérale et notamment de l'altération et de la genèse des argiles, de la dynamique de la matière organique, des transports de matiè- res dans le sol, enfin des principes généraux de la genèse et de l'évolution des sols. Cette première partie a subi de profondes modifications par rapport à la précédente édition. En particulier, le chapitre consacré à la dynamique des matières organiques a été entièrement refondu pour tenir compte de l'évolution rapide des connaissances dans ce domaine. Dans une moindre mesure, les chapitres consacrés au transport de matière, notamment aux complexes organo-métalliques, ainsi qu'aux principes généraux de la genèse (facteur temps et étude des cycles d'évolution) ont été également actualisés.

La deuxième partie discute et illustre le choix de la pédogenèse comme base de la classification des sols. Après une critique comparée des principaux systèmes taxonomiques, l'Auteur présente les grands types de sol de la classification française. Pour chacun d'entre eux, il adopte une structure de présentation spécifique, mais comportant toujours des données écologiques, morphologiques, les bases de la classification interne à chacune des classes et l'analyse des processus pédogénétiques majeurs. 